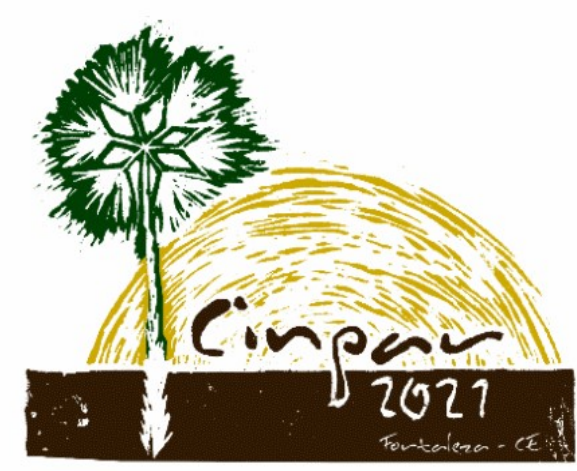

XVII Congresso Internacional sobre Patologia e Reabilitação das Construções

XVII Congreso Internacional sobre Patología y Rehabilitación de las Construcciones

XVII International Conference on Pathology and Constructions Rehabilitation

FORTALEZA (Brasil), 3 a 5 de junho de 2021

https://doi.org/10.4322/CINPAR.2021.017

\title{
UTILIZAÇÃO DE RESÍDUO DE PNEU COMO AGREGADO EM CONCRETO - ANÁLISE DE ENSAIOS MECÂNICOS
}

\author{
Use of Tire Waste as a Concrete Aggregate - Mechanical Test Analysis
}

André ROLOFF ${ }^{1}$, David Paim de MATOS ${ }^{2}$, Vitor Luis BRINKHUS ${ }^{3}$, Bárbara JORDANI ${ }^{4}$, David Brandão NUNES 5

\author{
${ }^{1}$ Faculdade FTEC, Novo Hambugo, Brasil, roloffandre@hotmail.com \\ 2 Faculdade FTEC, Novo Hambugo, Brasil, david.matos80@gmail.com \\ ${ }^{3}$ Faculdade FTEC, Novo Hambugo, Brasil, vlbrinkhus@gmail.com \\ ${ }^{4}$ Faculdade FTEC, Novo Hambugo, Brasil, bbjordani@gmail.com \\ ${ }^{5}$ Universidade do Vale do Rio dos Sinos, São Leopoldo, Brasil, davidbrnunes@gmail.com
}

\begin{abstract}
Resumo: Com a preocupação atual acerca da preservação do meio ambiente e de novas soluções para trabalharmos em prol da sustentabilidade, este estudo busca uma possibilidade benéfica para a natureza a adição de resíduos de pneu inservível para fabricação de concretos. A quantidade de borracha acumulada em todo o mundo tende a crescer, já que os pneus têm uma determinada vida útil e precisam ser descartados. Pensando nisso e para dar um destino eficaz para parte deste resíduo, foram realizados ensaios comparativos entre o Concreto com Adição de Borracha (CAB) - determinada a adição de $20 \%$ de borracha no total de agregado miúdo e o Concreto Referência (CR), buscando resultados que possam ajudar na sua implementação em grande escala na construção civil. Assim, com o intuito de avaliar os resultados obtidos na adição de borracha, foram realizados ensaios de resistência à compressão, tenacidade e massa específica (visando os concretos leves). A partir dos resultados encontrados foi possível verificar que os concretos CAB apresentaram menor resistência à compressão, em torno de $28,5 \%$ quando comparado aos concretos CR. A tenacidade encontrada no CAB foi maior, proporcionalmente, comparada ao CR. Portanto, a adição de resíduos de pneu para fabricação de concreto leve pode ser considerado satisfatório.
\end{abstract}

Palavras-chave: Concreto, Resíduo de Pneu, Agregado, Tenacidade, Sustentabilidade

\begin{abstract}
With the current concern about preserving the environment and new solutions for working towards sustainability, this study seeks a beneficial possibility for nature - the addition of waste tire for the manufacture of concrete. The amount of rubber accumulated worldwide tends to increase, as the tires have a certain useful life and need to be discarded. Thinking about it and to give an effective destination for part of this residue, comparative tests were carried out between Concrete with Rubber Addition (CAB) determined the addition of $20 \%$ rubber in the total of fine aggregate and the Concrete Reference (CR), seeking results that can help in its large-scale implementation in civil construction. Thus, in order to evaluate the results obtained in the addition of rubber, tests were performed on compressive strength, toughness and specific gravity (targeting light concretes). From the results found, it was possible to verify that $C A B$ concretes had less resistance to compression, around $28.5 \%$ when compared to CR concretes. The toughness found in the $C A B$ was proportionally greater, compared to the $C R$. Therefore, the addition of tire waste to the manufacture of lightweight concrete can be considered satisfactory.
\end{abstract}

Keywords: Concrete, Tire Waste, Aggregate, Tenacity, Sustainability 


\section{Introdução}

A retirada desenfreada de areia dos rios pode ocasionar problemas ambientais e modificar o habitat natural das espécies locais. A pesquisa de Ferreira et al., (2019) salienta que o setor da construção civil está diretamente ligado ao consumo mundial de areia, cobiçada pelas usinas de concreto que retiram diariamente elavadas quantidades desta matéria prima da natureza.

A elaboração de estudos para a reciclagem de materiais nocivos ao meio ambiente se faz constante, visando minimizar os impactos ambientais a nível mundial. Dentre os diversos resíduos possíveis, analisando seu grande impacto ambiental, temos a borracha proveniente de pneus inservíveis. A produção de pneus no Brasil chegou a 4,82 milhões no ano de 2020, de acordo com a Associação Nacional da Indústria de Pneumático. E são descartados pelo menos 450 mil toneladas de pneus por ano, o qual equivale a cerca de 90 milhões de unidades utilizadas em carros de passeio (FETRANSPAR).

Devido sua degradação ser muito lenta e este material estar descartado em elevadas quantidades, é muito conveniente estudarmos a adição da borracha na composição do concreto, visto que alguns autores (FAZZAN et al., 2016; SIMONETTI et al., 2020) vêm analisando as propriedades deste material e ressaltam sua viabilidade de uso para fabricação de concreto. As pesquisas realizadas tem demonstrado que o concreto com adição de borracha possui resistência mecânica inferior ao concreto convencional. Siddique e Naik (2004) explicam que este fenômeno pode ser atribuído à baixa aderência entre a borracha e a matriz de cimento.Em contrapartida, Simonetti et al., (2019) menciona que para manter a resistência com a adição do elastômero, é indispensavel elevar o consumo de cimento, ou seja, reduzir o fator água/cimento.

Dentro deste contexto, esta pesquisa tem como pilares a necessidade de preservar o meio ambiente e o desenvolvimento sustentável, visto que esse tipo de composto (água, areia, borracha, brita e cimento) contribui para uma forma de construção mais sustentável, diminuindo o consumo de recursos naturais e obtendo um material com características diferenciadas. Assim, este estudo busca analisar os efeitos da adição de borracha de pneu no concreto convencional sem função estrutural, avaliando sua resistência à compressão, tenacidade e massa específica.

\section{Materiais e Métodos}

A seguir serão descritos os materiais e os métodos utilizados na pesquisa.

\subsection{Materiais}

\subsection{1 - Cimento}

Foi utilizado um cimento Portland tipo CP IV-32, conforme a NBR 16697 (2018). Com adição de cinza pozolânica, resultante da queima de carvão em usinas termoelétricas, com teores compreendidos entre $15 \%$ e $50 \%$ da massa total. Sua massa específica é $2,82 \mathrm{~g} / \mathrm{cm}^{3}$.

\subsection{2 - Agregado Miúdo}

\subsubsection{1 - Areia}

O agregado miúdo natural empregado foi uma areia quartzosa, proveniente do Rio Jacuí, Rio Grande do Sul. A caracterização do agregado miúdo natural foi realizada conforme a norma NBR NM 248 (2003) para distribuição granulométrica e a NBR NM 45 (2006) para massa unitária. A massa unitária caracterizada é de $1,37 \mathrm{~g} / \mathrm{cm}^{3}$. A granulometria apresenta dimensão máxima de $6,3 \mathrm{~mm}$ e módulo de finura 3,53.

\subsubsection{2 - Borracha de pneu}


O resíduo de pneu inservível utilizado foi obtido na Renovadora de Pneus Caxiense - Rodovia BR 116, no 22.301, Km 153 na cidade de Caxias do sul/Rio Grande do Sul. A massa unitária é de 0,45 g/ $\mathrm{cm}^{3}$.

\subsection{3 - Agregado Graúdo}

O agregado graúdo natural empregado foi uma brita basáltica, proveniente de Caxias do Sul, Rio Grande do Sul. Obteve-se a distribuição granulométrica e a massa unitária de acordo com a NBR NM 248 (2003) e a NBR NM 45 (2006), respectivamente. A massa unitária é de $1,27 \mathrm{~g} / \mathrm{cm}^{3}$. A granulometria apresenta dimensão máxima de $25 \mathrm{~mm}$ e módulo de finura 7,81.

\subsection{Métodos}

\subsection{1 - Proporcionamento dos materiais}

Para a dosagem do Concreto com Adição de Borracha (CAB) e do Concreto Referência (CR) adotou-se o traço 1:2:3 nas proporções de cimento, areia e brita - com resistência estimada aos 28 dias entre $25-30 \mathrm{MPa}$. A adição de borracha triturada foi na proporção de $20 \%$ do agregado miúdo e a relação de água cimento foi de 0,61 .

\subsection{2 - Produção dos corpos de provas}

Os corpos de provas seguiram as recomendações da NBR 5738 (2015), cilíndricos, com dimensões de $10 \mathrm{~cm} x$ $20 \mathrm{~cm}$. Foram moldados seis corpos de prova (três do tipo CAB e três do tipo CR). A moldagem dos corpos de prova foi realizada de acordo a NBR 5738 (2015), e os corpos de prova foram submetidos à cura úmida, submersos em tanque com água e cal.

\subsection{3 - Resistência à compressão}

$\mathrm{Na}$ avaliação dos concretos quanto a resistência à compressão, para as amostras CAB1 e CR1 foi utilizada uma prensa hidráulica e para as amostras CAB2, CAB3, CR2 e CR3 os testes de tensão $x$ deformação foram realizados numa prensa eletromecânica EMIC com capacidade de $300 \mathrm{kN}$ (30000 kgf). O ensaio foi realizado conforme a NBR 5739 (2018), para cada tipo de concreto produzido foram ensaiados três corpos de prova cilíndricos na idade de 28 dias.

\subsection{4 - Tenacidade}

Para o cálculo da tenacidade das amostras foi gerado os gráficos Força x Deformação pelo software Tesc 400. Os resultados foram exportados para o programa Origin e, assim, foi calculada a área dos gráficos através de integral.

\subsection{5 - Massa específica}

A determinação da massa específica foi realizada com base na NBR 9778 (2005).

\section{Apresentação e Análise dos Resultados}

A seguir serão apresentados os resultados dos ensaios realizados, bem como as discussões. 


\subsection{Resistência à compressão}

Os gráficos a seguir, Figuras 1 e 2, apresentam as curvas típicas da relação Tensão x Deformação. Selecionaram-se as curvas das amostras CR3 e CAB3 considerando que o formato destas curvas pode ser extrapolado para as demais amostras, considerando que cada corpo de prova teve sua própria tensão máxima de ruptura. Estes valores estão descritos no Quadro 1.

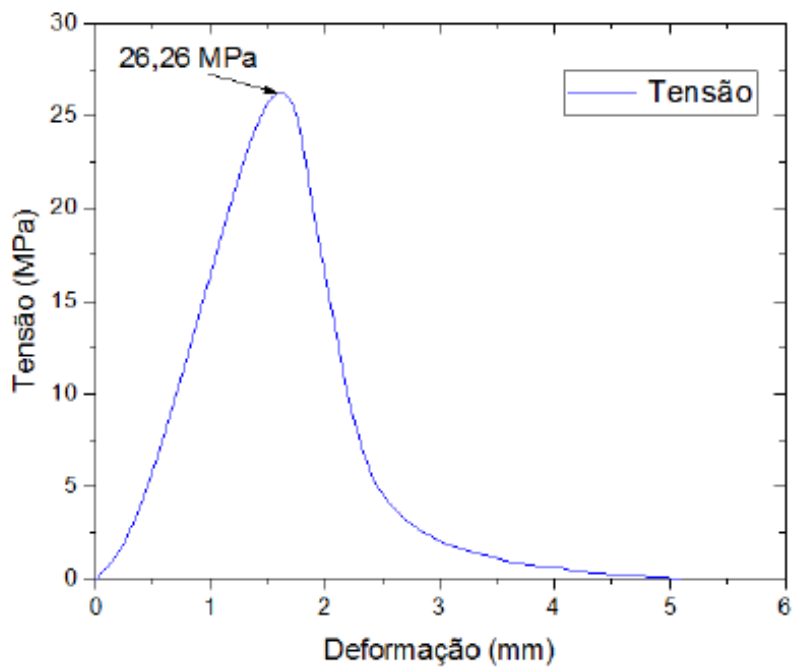

Figura 1 - Tensão x Deformação CR3

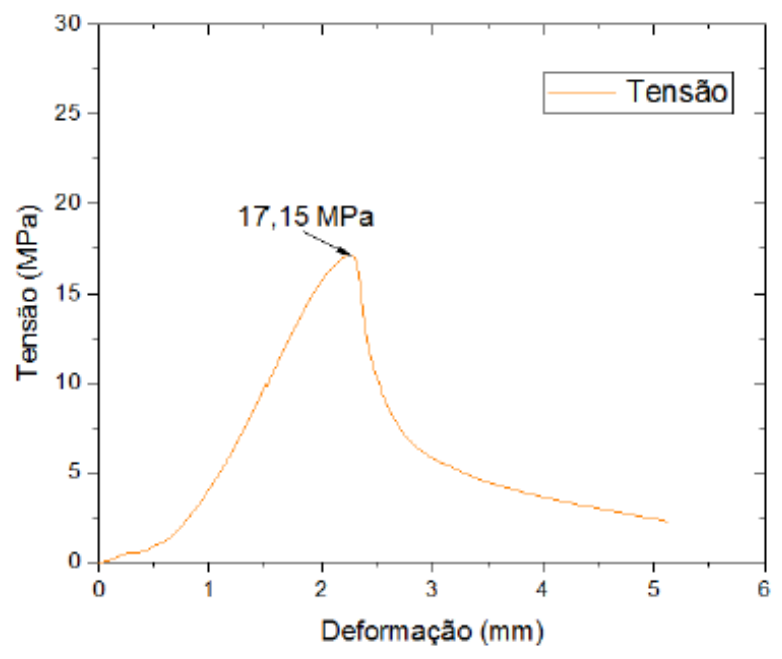

Figura 2 - Tensão x Deformação CAB3

Quadro 1 - Tensão de ruptura Concreto Referência e Concreto com Adição de Borracha

\begin{tabular}{|c|c|c|c|}
\hline Amostra & $\begin{array}{c}\text { Tensão de Ruptura } \\
\text { (MPa) }\end{array}$ & Amostra & $\begin{array}{c}\text { Tensão de Ruptura } \\
\text { (MPa) }\end{array}$ \\
\hline CR1 & 22,12 & Concreto CAB1 & 18 \\
\hline CR2 & 24,96 & Concreto CAB2 & 16,6 \\
\hline CR3 & 26,26 & Concreto CAB3 & 17,15 \\
\hline Média & 24,44 & Média & 17,25 \\
\hline
\end{tabular}

Através do quadro 1 é possível verificar os resultados obtidos no ensaio de ruptura O concreto CR1 apresentou tensão de ruptura de 22,12 MPa, já os concretos CR2 e CR3 apresentaram 24,96 MPa e CR3 26,26 $\mathrm{MPa}$, respectivamente. As amostras CAB1, CBA2 e CAB3 de concreto com adição de borracha apresentaram tensões de ruptura de $18 \mathrm{MPa}, 16,60 \mathrm{MPa}$ e 17,15 MPa, respectivamente. Para a avaliação final da resistência à compressão, foi calculada a média dos resultados encontrados na prensa hidráulica e na prensa eletromecânica EMIC, das três amostras de cada um dos compostos. $O$ concreto referência apresentou média de 24,44 MPa e o Concreto com Adição de Borracha de 17,25 MPa.

Analisando as médias encontradas, observa-se uma diminuição de $29,41 \%$ na resistência à compressão do CAB. Este resultado pode ser considerado satisfatório, pois Toutanji (1996) apresenta em sua pesquisa que a adição de resíduos de pneu no concreto causa uma perda de 25 à $30 \%$ em sua resistência à compressão. Esta queda na resistência pode ser explicada pelo estudo realizado por Turatsinze, Bonnet e Granju (2005), que ao analisarem a microestrutura de concretos com resíduos de borracha de pneu, observaram uma zona de transição pasta de cimento-borracha fraca quando comparada com a pasta de cimento-areia. Estes autores afirmam ainda que essa microestrutura em particular seria um fator adicional para a ocorrência da diminuição da resistência à compressão dos concretos quando adicionados de borracha. Nos ensaios de compressão, Silva et al., (2017) observou que as amostras sem adição de borracha, ao se 
romperem, se esfacelaram especialmente nas bordas, enquanto os blocos contendo granulado de borracha não esfacelaram. Essa característica foi atribuída à presença da borracha, a qual possui flexibilidade e capacidade de alongar e girar em torno de seus eixos. Em estudos realizados por Alves (2020), os concretos com substituição parcial de agregado miúdo natural por borracha de pneu não alcançaram a resistência mínima de $20 \mathrm{MPa}$, isto pode ter acontecido por influência de uma combinação complexa de valores como o teor de substituição (12,5\% e $15 \%)$, granulometria dos resíduos, aumento na incorporação de ar e enfraquecimento da zona de transição.

\subsection{Tenacidade}

Para análise quanto à tenacidade foi utilizado o software Ogirin, calculando a área dos gráficos das amostras CR2, CR3, CAB2 e CAB3, figuras 3, 4, 5 e 6, respectivamente. $O$ Quadro 2 apresenta os resultados encontrados pelo software.

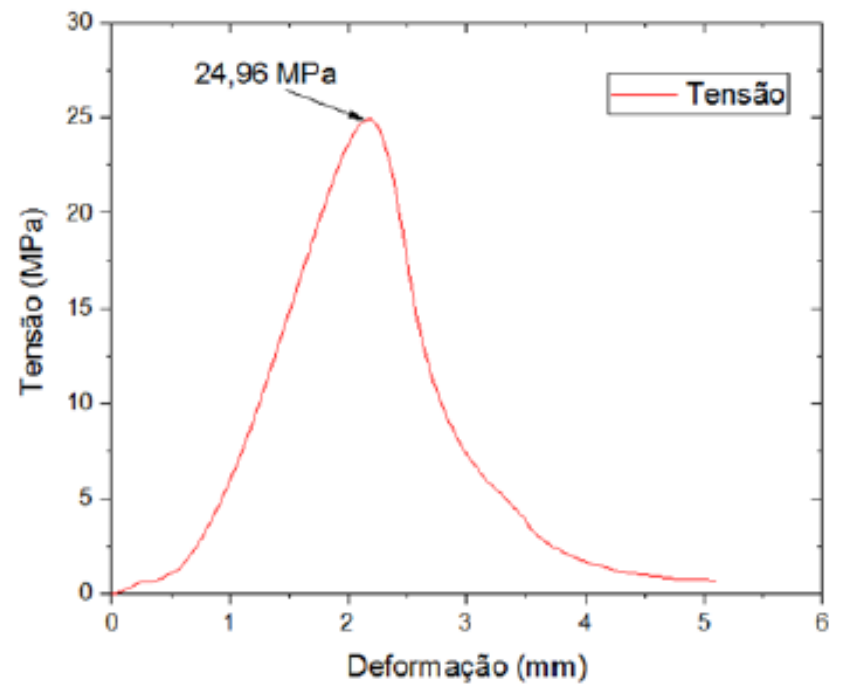

Figura 3 - Tensão x Deformação CR2

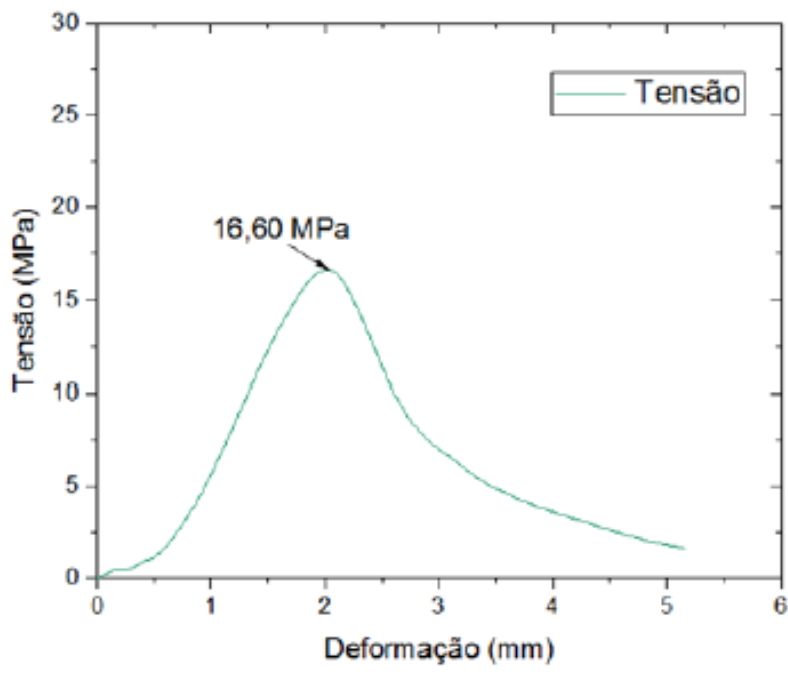

Figura 5 - Tensão x Deformação CAB2

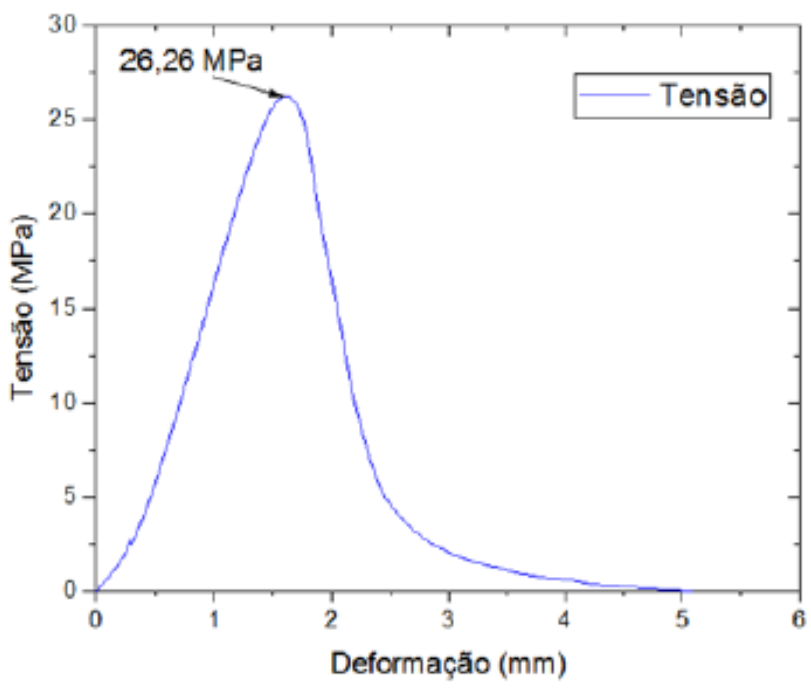

Figura 4 - Tensão x Deformação CR3

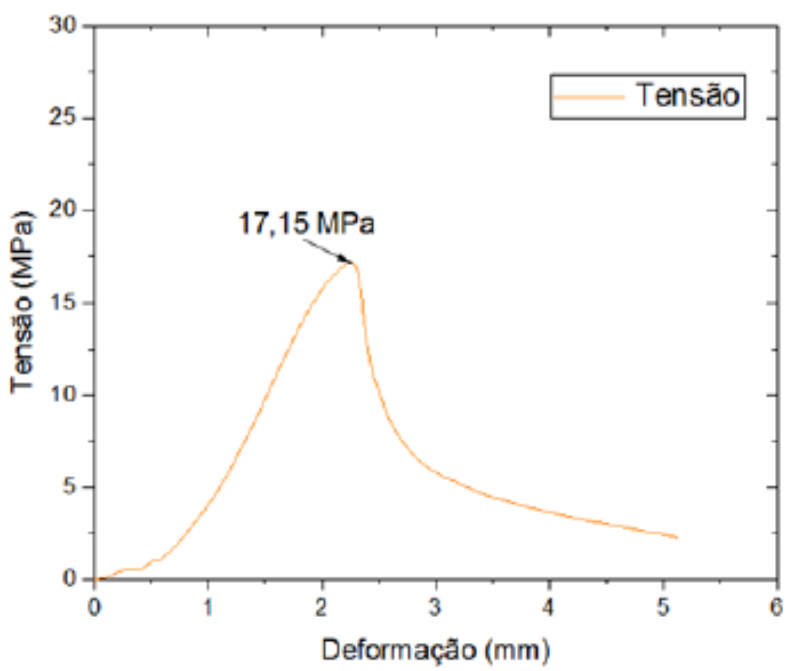

Figura 6 - Tensão x Deformação CAB3 
Quadro 2 - Tensão de ruptura Concreto Referência e Concreto com Adição de Borracha

\begin{tabular}{|c|c|c|c|}
\hline CR2 & CR3 & CAB2 & CAB3 \\
\hline$x 1($ Beginning $X)=0$ & $x 1$ (Beginning $X)=0$ & $x 1$ (Beginning $X)=0$ & $x 1($ Beginning $X)=0$ \\
\hline$x 2($ Ending $X)=5,1071$ & $x 2$ (Ending $X)=5,0873$ & $x 2($ Ending $X)=5,1639$ & $x 2($ Ending $X)=5,1235$ \\
\hline $\begin{array}{l}\mathrm{i} 1 \text { (Index for Beginning } \\
\mathrm{X})=1\end{array}$ & $\begin{array}{l}\text { i1(Index for Beginning } \\
X)=1\end{array}$ & $\begin{array}{l}\text { i1(Index for Beginning } X) \\
=1\end{array}$ & $\begin{array}{l}\text { i1(Index for Beginning } X) \\
=1\end{array}$ \\
\hline $\begin{array}{l}i 2(\text { Index for Ending } X)= \\
477\end{array}$ & $\begin{array}{l}\text { i2(Index for Ending X) } \\
=427\end{array}$ & $\begin{array}{l}\text { i2 }(\text { Index for Ending } X)= \\
427\end{array}$ & $\begin{array}{l}\text { i2 }(\text { Index for Ending } X)= \\
455\end{array}$ \\
\hline $\begin{array}{l}\text { area(Area of the } \\
\text { Integration) }=38,88\end{array}$ & $\begin{array}{l}\text { area(Area of the } \\
\text { Integration) }=36,78\end{array}$ & \begin{tabular}{|l|} 
area(Area of the \\
Integration) $=33,37$ \\
\end{tabular} & \begin{tabular}{|l|} 
area(Area of the \\
Integration) $=30,29$ \\
\end{tabular} \\
\hline $\begin{array}{l}\text { y0 (Maximum Height })= \\
24,96\end{array}$ & $\begin{array}{l}\text { y0(Maximum Height) } \\
=26,25\end{array}$ & $\begin{array}{l}\text { y0(Maximum Height) }= \\
16,60\end{array}$ & $\begin{array}{l}\text { y0(Maximum Height) }= \\
17,16\end{array}$ \\
\hline $\begin{array}{l}x 0(X \text { Value in Maximum } \\
\text { Height })=2,18\end{array}$ & $\begin{array}{ll}\mathrm{x} 0(\mathrm{X} \quad \text { Value in } \\
\text { Maximum Height })= \\
1,61\end{array}$ & $\begin{array}{l}x O(X \text { Value in Maximum } \\
\text { Height })=2,02\end{array}$ & $\begin{array}{l}x 0(X \text { Value in Maximum } \\
\text { Height })=2,24\end{array}$ \\
\hline $\begin{array}{l}\mathrm{dx}(\text { Peak Width at Half } \\
\text { Height })=1,28\end{array}$ & $\begin{array}{l}\mathrm{dx}(\text { Peak Width at Half } \\
\text { Height })=1,25\end{array}$ & $\begin{array}{l}\mathrm{dx}(\text { Peak Width at Half } \\
\text { Height })=1,58\end{array}$ & $\begin{array}{l}\mathrm{dx}(\text { Peak Width at Half } \\
\text { Height })=1,18\end{array}$ \\
\hline
\end{tabular}

Os valores em negrito no Quadro 2 são as áreas encontradas e, consequentemente, as respectivas tenacidades. Para calcular a relação da tenacidade entre o Concreto Referência e o Concreto com Adição de Borracha, fez-se a razão entre: área do gráfico x tensão. Para este cálculo da razão, obteve-se a média das áreas dos gráficos de cada tipo de concreto, conforme Equação 1 e 2.

$C R=\frac{38,88+36,78}{2}=37,83 \quad($ Equação 1$)$

$C A B=\frac{33,36+30,29}{2}=31,82 \quad($ Equação 2)

Desta forma, a razão entre a área do gráfico e tensão são apresentadas nas Equações 3 e 4.

$C R=\frac{37,83}{24,13}=1,567 \quad$ (Equação 3)

$C A B=\frac{31,82}{17,25}=1,844 \quad$ (Equação 4)

Sendo assim, o concreto $C A B$ apresentou o valor de 1,844 para a razão entre a tenacidde total e a tensão, maior que o concreto CR que resultou em uma razão de 1,567. Segundo Li (2004) os concretos com adição de resíduos de borracha de pneu apresentam, quando comparados aos concretos sem adição, um aumento no valor de sua tenacidade (área sob a curva). O resultado é satisfatório, pois a adição de resíduos de pneu inservível foi positiva quando analisada a tenacidade das amostras.

\subsection{Massa específica}

Conforme Almeida (2002) a massa específica do concreto depende de vários fatores, principalmente da natureza dos agregados, da granulométrica e do método de compactação empregado. As massas específicas foram calculadas de acordo com a NBR 9833 (2008) e adotou-se o volume do corpo de prova igual a $0,0016 \mathrm{~m}^{3}$. Calcularam-se as médias das massas específicas e obteve-se um decréscimo de $3,75 \%$ nos concretos com adição de pneu inservível, o que já era esperado. Neville (1997) menciona que o peso próprio dos elementos de concreto, pode apresentar uma grande proporção de carga a uma estrutura. Portanto, o uso de concretos com massa específica reduzida pode resultar vantagens significativas quanto a elementos estruturais com menor seção transversal e uma correspondente diminuição das dimensões das fundações. A utilização de borracha reciclada de pneus como agregado para produção de concreto leve contribui para a resolução de um dos problemas ambientais mais cruciais, a deposição dos resíduos sólidos. 


\section{Conclusões}

Após o estudo realizado, foi possível verificar que a adição de borracha reduziu a resistência à compressão, mas com resultados foram positivos, visto que se mantiveram entre 25 a $30 \%$ abaixo em relação ao concreto referência. $O$ parametro de tenacidade obtido com a adição de borracha manteve a linha das pesquisas atuais sobre o assunto, as quais indicam um aumento desta característica nos concretos CAB.

Salienta-se que a razão entre as áreas dos gráficos Tensão x Deformação e as tensões (MPa) encontradas foi maior nos Concretos com Adição de Borracha, podendo se considerar um sucesso. Para os resultados encontrados refrente a massa específica do CAB foi 3,75\% menor que o CR, confirmando o que era previsto, pois à borracha é mais leve que a areia. Cabe destacar que este parametro pode ser levado em consideração na fabricação de concretos leves na construção civil, como alternativa sustentável e barata.

Portanto, o estudo da adição de resíduos de pneu inservível foi positivo, indicando que é possível reaproveitar este resíduo ao final de sua vida útil. O trabalho desenvolvido mostra-se eficaz para a utilização em peças de concreto para pavimentação sendo necessário desenvolver maiores estudos quanto à resistência à abrasão e absorção de água, conforme NBR 9780 (1987) e NBR 9781 (2013).

\section{Referências Bibliográficas}

ALMEIDA, L. C. Concreto. Campinas, 2002. (Apostila).

ALVES, Camila dos Reis. Uberlândia, 2020. Avaliação do comportamento hidrotérmico de concretos produzidos com residuos de borracha de pneu e poliestireno expandido para aplicação em paredes de concreto. p. 1-201. Disponível em: http://doi.org/10.14393/ufu.di.2020.358

ASSOCIAÇÃO BRASILEIRA DE NORMAS TÉCNICAS - ABNT. NBR 16697: Cimento Portland - Requisitos. ABNT. Rio de Janeiro, 2018.

ASSOCIAÇÃO BRASILEIRA DE NORMAS TÉCNICAS. NBR NM 45: Agregados - Determinação da massa unitária e do volume de vazios. Rio de Janeiro, 2006.

ASSOCIAÇÃO BRASILEIRA DE NORMAS TÉCNICAS. NBR 5738: Concreto - Procedimento para moldagem e umidade de corpos-de-prova. Rio de Janeiro, 2015.

ASSOCIAÇÃO BRASILEIRA DE NORMAS TÉCNICAS. NBR 5739: Concreto - Ensaio de compressão de corpos-deprova cilíndricos. Rio de Janeiro, 2018.

ASSOCIAÇÃO BRASILEIRA DE NORMAS TÉCNICAS. NBR 9833: Concreto fresco - Determinação da massa específica e do teor de ar pelo método gravimétrico. Rio de Janeiro, 2008.

ASSOCIAÇÃO BRASILEIRA DE NORMAS TÉCNICAS. NBR NM 248: Agregados - Determinação da composição granulométrica. Rio de Janeiro, 2003.

FAZZAN, João Victor et al. Estudo da viabilidade de utilização do Resíduo de Borracha de Pneu em Concretos Estruturais. Periódico Eletrônico "Fórum Ambiental da Alta Paulista" , [s. I.], v. 12, ed. 6, p. 118, 132, 2016. DOI http://dx.doi.org/10.17271/1980082712620161484. Disponível em: https://www.amigosdanatureza.org.br/publicacoes/index.php/forum_ambiental/article/view/1484. Acesso em: 5 jul. 2020.

FETRANSPAR (Paraná). Cerca de 450 mil toneladas de pneus são descartados por ano no Brasil. In: Cerca de 450 mil toneladas de pneus são descartados por ano no Brasil. Paraná: Fetranspar / Sest Senat, 17 fev. 2017. Disponível em: https://www.fetranspar.org.br/giro-pelo-setor/cerca-de-450-mil-toneladas-de-pneus-saodescartados-por-ano-no-brasil/. Acesso em: 5 jul. 2020.

FERREIRA, R.L.S. et al. Efeitos do uso de areia de praia nas propriedades de argamassas mistas: análise da variação granulométrica. Matéria (Rio J.) vol.24 no.2 Rio de Janeiro, 2019.

LI, G. et al. Waste tire fiber modified concrete. Composites, part B: engineering, v. pat B 35, p.305-312, 2004. NEVILLE, Adam Matthew. (Trad) Salvador E. Giammusso. Propriedades do concreto. 2 ed. ver. Atual. São Paulo: Pini, 1997.

SIMONETTI, C.; PEREIRA, J. W. ; TUTIKIAN, B. F. . Aproveitamento de borracha reciclada de pneu inservível na construção civil. In: $11^{\circ}$ Fórum Internacional de Resíduos Sólidos (11FIRS), 2020, Online. Anais do 11FIRS, 2020. 
SIMONETTI, C.; BAUER, A.L. ; GIL, A.M. ; TUTIKIAN, B.F. . Análise das Propriedades Físicas e Mecânicas de Concretos com Utilização de Resíduos de Pneus Inservíveis. In: 61 CBC, 2019, Fortaleza. Anais do 61 Congresso Brasileiro do Concreto, 2019.

SILVA, Thayane Dias et al. Uso de granulado de borracha em substituição parcial ao agregado miúdo na produção de tijolos ecológicos. Revista Matéria (Rio de Janeiro), Rio de Janeiro, v. 22, ed. 4 , 2 jan. 2017. DOI https://doi.org/10.1590/s1517-707620170004.0239.

Disponível em:

https://www.scielo.br/scielo.php?script=sci_arttext\&pid=S1517-70762017000400428\&lng=pt\&tlng=pt.

Acesso em: 6 jul. 2020.

TOUTANJI, H. A. The use of rubber tire particles in concrete to replace mineral aggregates. Cement \& Concrete Composites, v.18, p.135-139, 1996.

TURATSINZE, A.; BONNET, S.; GRANJU, J. L. Mechanical Characterisation of Cement-Based Mortar Incorporating Rubber Aggregates From Recycled Worn Tyres. Building and Environment,v. 40, n. 2, p. 221226, 2005. 